\title{
Kernos
}

Revue internationale et pluridisciplinaire de religion grecque antique

$17 \mid 2004$

Varia

\section{José Carlos BERMEJO BARRERA, Fátima DÍEZ PLATAS, Lecturas del mito griego}

\section{María Carmen Barrígon Fuentes}

URL : http://journals.openedition.org/kernos/1496

DOI : 10.4000/kernos. 1496

ISSN : 2034-7871

\section{Éditeur}

Centre international d'étude de la religion grecque antique

Édition imprimée

Date de publication : 1 janvier 2004

ISSN : 0776-3824

\section{Référence électronique}

María Carmen Barrígon Fuentes, « José Carlos bermejo barRera, Fátima díEZ platas, Lecturas del mito griego », Kernos [En ligne], 17 | 2004, mis en ligne le 16 juin 2011, consulté le 24 septembre 2020. URL http://journals.openedition.org/kernos/1496 ; DOI : https://doi.org/10.4000/kernos.1496 
José Carlos Bermejo Barrera, Fátima Díez Platas, lecturas del mito griego, Madrid, Ediciones Akal, 2002. 1 vol. $13,5 \times 22 \mathrm{~cm}, 329$ p. (Akal Universitari. Serie Interdisciplinar). ISBN : 84-460-1540-4.

Les seize travaux que comprend ce livre sont le fruit de la recherche de deux historiens du monde antique de l'Université de St Jacques de Compostelle qui approchent le mythe grec à partir d'une double perspective, théorique et historique.

Le contenu est organisé en deux parties bien différenciées. La première partie, rédigée par José Carlos Bermejo, réunit neuf articles déjà publiés, à l'exception de deux. On connaît bien la trajectoire de recherche de l'A. par rapport au mythe avec des titres tels que Introduction à la sociologie du mythe grec (1979), Wythe et parenté dans la Grèce archaique (1980), ou Mythologie et mythes de l'Espagne préromaine (1982-1986), dans lesquels il effectue des recherches de caractère méthodologique sur la base de modèles anthropologiques, sociologiques et psychologiques. C'est dans cette ligne qu'il poursuit ses travaux actuels, fruits d'une profonde réflexion sur quelques-unes des tendances à partir desquelles le mythe grec a été analysé. Il convient de signaler trois caractéristiques : la minutie de l'analyse, la clarté de l'exposition et la simplicité du langage.

L'A. consacre les deux premiers articles (p. 7-51) à établir une série d'orientations méthodologiques sur l'étude de la religion grecque. Il défend la comparaison dans l'Histoire des religions à travers une révision critique des courants les plus remarquables, tout en prévenant des clangers, et il insiste sur le fait qu'il ne faut pas oublier que la réalité historique est seulement accessible partiellement à travers une documentation fragmentaire. Dans le deuxième travail, publié préalablement en anglais dans la revue Dialogues d'bistoire ancienne (16 [1990], p. 215-230), il pose le problème de l'étude archéologique de la religion. Dans un exposé clair et minutieux, il met en relief le fait que, dans le domaine de l'archéologie, il s'agit d'établir les liens existants entre les objets et leurs fonctions culturelles et symboliques; c'est pourquoi il est plus prudent d'éviter le terme de religion dans les cas où l'archéologie devient l'unique source possible de connaissance historique, surtout quand, aussi bien en histoire qu'en archéologie, on succombe habituellement à la tentation de confondre inconsciemment la documentation conservée avec la réalité historique à étudier.

Dans les sept autres articles (p. 52-165), il fait une lecture du mythe selon des perspectives différentes : historique, anthropologique et sociologique. Il y inclut des exemples concrets comme celui d'Artémis, lorsqu'il met en relation mythe et méthode historique, faisant une exposition exhaustive des interprétations de "l'Ecole dite de Rome ", ou l'exemple des figures de Typhon et d'Hécate, à travers lesquelles il analyse les possibilités de lecture offertes par la Théogonie hésiodique.

L'A. pense qu'il faut reformuler non seulement notre conception du pouvoir, mais aussi nos idées du réel et de l'idéal en histoire. Ceci l'amène à reposer le problème de la relation entre mythe et polis, puisqu'il considère que le mythe fait partie de la réalité et que la polis n'est pas une réalité univoque, mais plurielle. Il analyse les théories de Leopold von Ranke sur l'histoire et le mythe, conteste sa conception du réel et de l'idéal en histoire et défend un renouvellement de ces études.

Il nous offre également un rapprochement de caractère sociologique du mythe grec dans ses relations avec la littérature, en mettant en valeur les traits les plus génériques qui configurent leur dialogue. L'A. aborde une vision méthodique où il essaie d'indiquer la validité, d'une part, de la notion de «jeu de langage ", qui avait déjà été défendue par Ludwig Wittgenstein dans ses Recherches philosophiques, et d'autre part, de la notion de "cercle herméneutique » défendue par Hans-Georg 
Gadamer et son école, comme instruments pour l'analyse du mythe et de ses représentations littéraires.

L'expérience remarquable de recherche de cet auteur est appréciée tout au long de ces pages où, à aucun moment, il ne se laisse entraîner par sa formation d'historien, mais essaie de montrer au lecteur les chemins qui conduisent aux différentes lectures du mythe grec. Cependant, on peut déplorer l'absence d'une introduction à ces neuf travaux (peut-être aussi de quelques conclusions ponctuelles servant de synthèse). Il aurait été bien utile d'avoir une sélection bibliographique des travaux les plus éminents qui servirait de mise à jour dans ce domaine de recherche.

La seconde partie (p, 169-328), due à Fátima Díaz Platas, est la publication d'une thèse de doctorat soutenue à l'Université Complutense de Madrid, en 1996, sous la direction des professeurs Alberto Bernabé et Ricardo Olmos. L'A. analyse, à travers les sources littéraires d'époque archaïque, les possibilités de lecture offertes par la figure des Nymphes, un «ensemble » mythique dont le culte s'est propagé pendant toute l'Antiquité. Elles fréquentaient les espaces ruraux et urbains et ont même survécu dans le cadre chrétien en devenant des personnages de la mythologie populaire.

L'A. organise la matière en sept chapitres. Dans les deux premiers, elle analyse le terme de nymphè et l'usage que les textes grecs en font et qui renvoient à différents concepts. Le troisième chapitre est consacré aux diverses apparitions que font les Nymphes dans la littérature grecque archäque, tandis que dans les autres chapitres elle parle du thème de l'origine et de la généalogie de ces divinités, du cadre naturel où elles habitent et de la relation qu'elles entretiennent avec différents dieux. Elle met en relief leur rôle insignifiant dans les récits mythiques, excepté le mythe de Persée.

Le choix de l'A. de distribuer le travail en articles indépendants ne nous paraît pas approprié, puisqu'elle utilise le même matériel qui apparât dans sa thèse sans aucune modification. À notre avis, il eût été plus pertinent de commencer par une introduction dans laquelle eît été présentée la gestation de la recherche, car ce livre a été publié cinq ans après sa soutenance. Elle aurait pu terminer sur des conclusions et compléter le tout par une bibliographie bien choisie, d'autant plus qu'en 2001, Jennifer Larson a publié chez Oxford une étude exhaustive sur les Nymphes dans la Grèce antique (Greek Nymphs. Myth, Cult, Lore), à laquelle nous ne trouvons aucune référence.

En résumé, nous sommes devant un livre qui ouvre de nouveaux chemins pour l'étude du mythe et qui représente, dans le large éventail de perspectives de rapprochement du mythe, une phase de réflexion critique. Il pose également des problèmes débattus de mythologie sur la base d'une analyse détaillée de témoignages littéraires. Les précisions que nous avons signalées n'enlèvent rien à la qualité de cet ouvrage.

Carmen Barrigón (Universidad de Valladolid)

Synnøve Des BOUVRIE (éd.), Myth and Symbol I. Symbolic phenomena in ancient Greek culture. Papers from the first international symposium on symbolism at the University of Tromsø, June 4-7, 1998, Bergen, 2002. 1 vol. $17,5 \times 24 \mathrm{~cm}, 332 \mathrm{p}$. (Papers from the Norwegian Institute at Athens, 5). ISBN : 82-91626-21-9.

Si l'on en croit le titre de ce volume, le mythe est ici traité en tant que phénomène symbolique. C'est essentiellement dans la substantielle introduction (plus de cinquante pages) de l'éditrice que cette dimension est abordée. Elle part en quête d'une définition du mythe - le problème récurrent de ce type d'étude - en situant son propos au 